\title{
Correction to: In Memory of Dr. Jean-Robert Bonami
}

\author{
Zheng-Li Shi ${ }^{1}$ (I)
}

Published online: 28 October 2021

(C) Wuhan Institute of Virology, CAS 2021

\section{Correction to: Virologica Sinica}

https://doi.org/10.1007/s12250-021-00456-x

The title of this obituary should be "In Memory of Dr. Jean-Robert Bonami". The figure legend should be "Photo of Dr. Bonami”.

The original article can be found online at https:// doi.org/10.1007/s12250-021-00456-x.

\footnotetext{
Zheng-Li Shi

zlshi@wh.iov.cn

1 Wuhan Institute of Virology, Chinese Academy of Sciences, Wuhan 430071, China
} 